\title{
Synthesis of Hydroxyapatite Nanoparticles in Presence of a Linear Polysaccharide
}

\author{
Humberto A. Monreal Romero, ${ }^{1}$ José Mora Ruacho, ${ }^{2}$ \\ Carlos A. Martínez Pérez, ${ }^{3}$ and Perla E. García Casillas ${ }^{3}$ \\ ${ }^{1}$ Facultad de Odontología, U.A.CH., Ciudad Universitaria Campus I, 31000 Chihuahua, CHIH, Mexico \\ ${ }^{2}$ Facultad de Ingeniería, U.A.CH., Circuito No. 1, Campus Universitario 2, 31125 Chihuahua, CHIH, Mexico \\ ${ }^{3}$ Instituto de Ingeniería y Tecnología, U.A.C.J., Avenida del Charro No. 610 Norte, Ciudad Juárez, CHIH, Mexico
}

Correspondence should be addressed to Humberto A. Monreal Romero; hmonreal@uach.mx

Received 17 December 2012; Revised 31 March 2013; Accepted 4 April 2013

Academic Editor: Guoping Chen

Copyright (C) 2013 Humberto A. Monreal Romero et al. This is an open access article distributed under the Creative Commons Attribution License, which permits unrestricted use, distribution, and reproduction in any medium, provided the original work is properly cited.

\begin{abstract}
Hydroxyapatite nanoparticles compounds were synthesized. Natural hydroxyapatite and a linear polysaccharide (1-3 linked $\beta$ - $D$ galactopyranose and 1,4 linked 3,6 anhydro- $\alpha$-L-galactopyranose) were used as a precursor in its formation. Our purpose was to produce nanoparticles in the presence of a linear polysaccharide with the use of a gelification method. The powder sample was evaluated by scanning tunneling microscope (STM), Brunauer-Emmett-Teller (BET) analysis, X-ray diffraction pattern (XRD), differential thermal analysis (DTA), infrared (IR) analysis, and thermal gravimetric analysis (TGA). According to the results, it was found that these nanoparticles can successfully be synthesized using a polysaccharide in a solution. On the other hand, the XRD peak intensity corresponds to hydroxyapatite structure in the range of temperature of $810^{\circ} \mathrm{C}$. The influence of the polysaccharide on the evolution of the nanoparticles has been demonstrated. This observation opens up new routes for the fabrication of nanoparticles using polysaccharides network. The synthesized nanoparticles have diameters ranging from $10 \mathrm{~nm}$ to $11 \mathrm{~nm}$ approximately. The elaboration conditions such as $\mathrm{pH}$ and concentration were optimized in this solution.
\end{abstract}

\section{Introduction}

Hydroxyapatite (HA) is a kind of material that constitutes hard tissues like bone and teeth. This material exhibits characteristics such as low solubility in water or body fluids and can be very attractive for several applications such as bioceramic in the field of medicine or industrial catalysis [1]. Also, with hydroxyapatite different methods of production of particles can be used and with these methods, several structures of different materials can be synthesized, such as nanoparticles, rods, and needles [2, 3]. Several materials have been used in the past with a progressive increase in the number of therapeutic applications; among these complex products are the nanoparticles for obtaining drugs such as liposomes and diagnostics agents $[4,5]$. One of the most interesting self-assembly processes is related to the exposition of hydroxyapatite (HA) to several metallic precursors as titanium, aluminum, and niobium for the synthesis of coating agents $[6,7]$. Nanoparticles of HA also have been studied for the synthesis of biocrystals using microwave irradiation [8]. Others examples are in the formation of nanostructures that use HA nanoparticles by means of aqueous solution/TX$100 / n$ butanol/cyclohexane and cetyltrimethylammonium bromide (CTAB) [9]; these structures can be synthesized with yttrium [10], linear polysaccharide (Hyaluronic acid) [11], and poly (vinyl alcohol) (PVA) [12], among others. The need of forming new components to nanometric scale has been made possible to explore new methods such as nanoemulsions [13], sol-gel [14], and mechanical activation [15]. In the present work we have synthesized HA nanoparticles facilitated by a linear polysaccharide such as $\beta-D$ galactopyranose and 1,4 linked 3,6 anhydro- $\alpha$-L-galactopyranose in order to promote a controlled growth of spherical nanoparticles. 


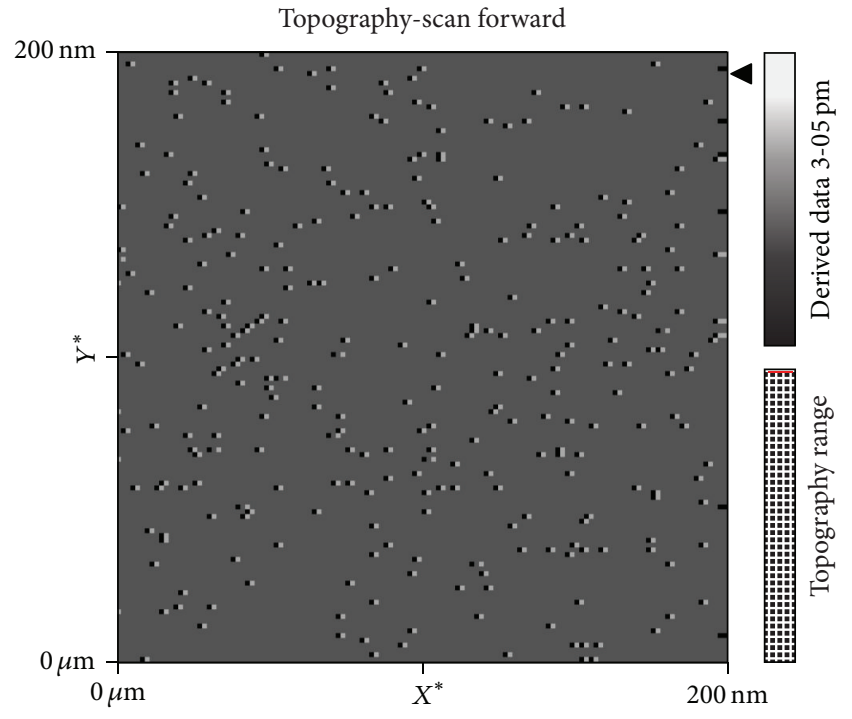

FIgURE 1: Image compounds of HA in STM.

\section{Experimental}

2.1. Synthesis of HA Nanoparticles. The HA nanoparticles were made as described hereafter. The compounds were prepared with the addition of $20 \mathrm{~g}$ of natural hydroxyapatite $3 \mathrm{Ca}_{3}\left(\mathrm{PO}_{4}\right)_{2} \mathrm{Ca}(\mathrm{OH})_{2}$ powder in a solution consisting of $200 \mathrm{~mL}$ of ethanol and $50 \mathrm{~mL}$ of acetic acid. Then a solution of repeated units of $99 \%$ chemically pure grade polysaccharide (1-3 linked $\beta-D$ galactopyranose and 1, 4 linked 3, 6 anhydro$\alpha$-L-galactopyranose) was used and added to this solution for gel formation.

Approximately $200 \mu \mathrm{L}$ of the solution of polysaccharide $(0.8 \%)$ was warmed up to $30^{\circ} \mathrm{C}$ for about 30 minutes. In this way, the hydroxyapatite growth was controlled by the polysaccharide network. Later, the gel was placed in $1 \mathrm{~mL}$ tube and centrifuged at $12,000 \mathrm{rpm}$ for 5 minutes at $28^{\circ} \mathrm{C}$. Afterwards, the concentrated gel was poured off and the precipitate was washed several times with deionized water to eliminate any amount of gel residue. Further centrifugal action to $12,000 \mathrm{rpm}$ for 5 minutes was carried out to recover the powder precipitate, followed by a 48-hour period in an incubator at $28^{\circ} \mathrm{C}$ to evaporate any residual water. After this, the powders were calcined in a laboratory muffle at $810^{\circ} \mathrm{C}$ for 2 hours. Afterwards, to characterize the compounds, a scanning tunneling microscope equipped with $\mathrm{Pt} / \mathrm{Ir}$ tips (BT00400) was used and the images were processed using a software version 1-6-0-0. Also the HA nanoparticles were processed in a simultaneous thermal analyzer DTA-TGA TA instruments at a heating rate of $10^{\circ} \mathrm{C} / \mathrm{min}$ in air and the crystalline phase of powders was identified by X-ray diffraction pattern (XRD) using a $\mathrm{CuK}(\alpha)$ source at $0.1542 \mathrm{~nm}$ in a Phillips X'PERT X-Ray diffractometer. The surface area analysis was measured for Brunauer-Emmett-Teller analysis (BET). Infrared spectra of the samples were analyzed using Perkin-Elmer infrared spectrophotometers.

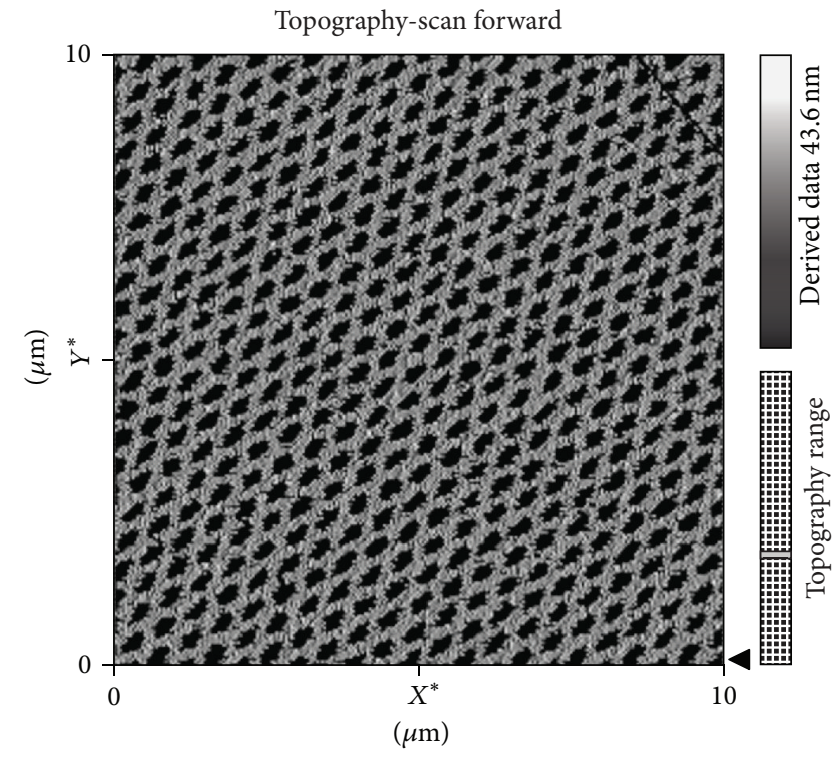

FIGURE 2: Image of polysaccharide network in STM.

\section{Results and Discussion}

The morphology of the compounds was investigated by scanning tunneling microscope (STM). Figure 1 shows the image in STM of nanoparticles of 10-11 nm in diameter. The entire surface is covered with nanoparticles; most of them are rather homogenous. This image suggests that the electrical behavior of the tunneling junction composed of HA nanoparticles is dominated by an electron-transfer mechanism characteristic of isolated nanoparticles. The process of formation of nanoparticles indicated by the STM image is a kinematically controlled process. In this way, the system evolves and organizes in such a state or nanostructure that is consistent with the lowering of the total free energy of the system, including the free energies present in volume as well as free energies present in interface. The energies can be elastic strain energies due to the misfit between the various phases as well as between the actual system and the substrate. The interface energy leads to the selection of those surfaces and interfaces that have the lowest energies.

In this context, upon addition of the polysaccharide, and once the solution has been mixed, the gelation process begins (this is the so-called gelation point). From this point, the conversion sol-gel is gradual and increasing. Particles become interconnected in the polysaccharide network, as shown in Figure 2.

For this reason, one of the most important properties of nanoparticles is their ability to remain in the network of the polysaccharide. This suggests the possibility that the hydroxyapatite has a high affinity for the polysaccharide and therefore would be more effectively retained in the network serving as a mold system to the synthesis of nanomaterials. Also, the reason for using the polysaccharide network is to control the shape and size of the particles.

Figure 3 shows a current in cross-section on unique scanning speed. High current spikes are generated at fast scanning 


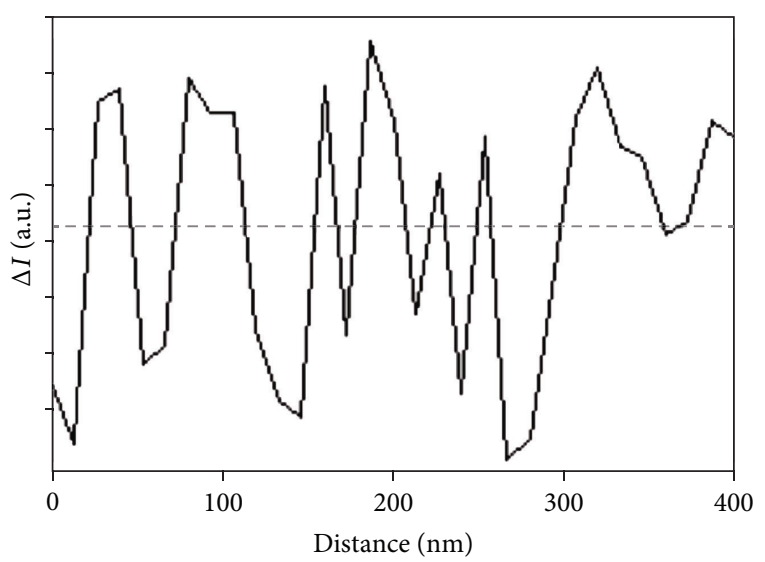

FIgURE 3: Spectroscopy by scanning tunneling microscopy.

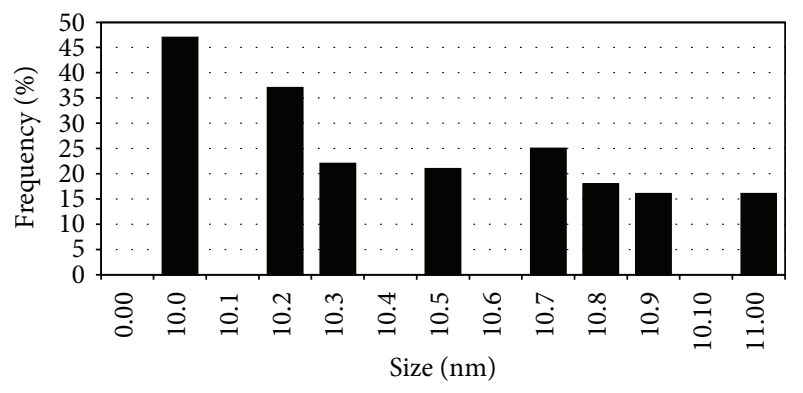

FIgURE 4: Particle size distribution of HA nanoparticles.

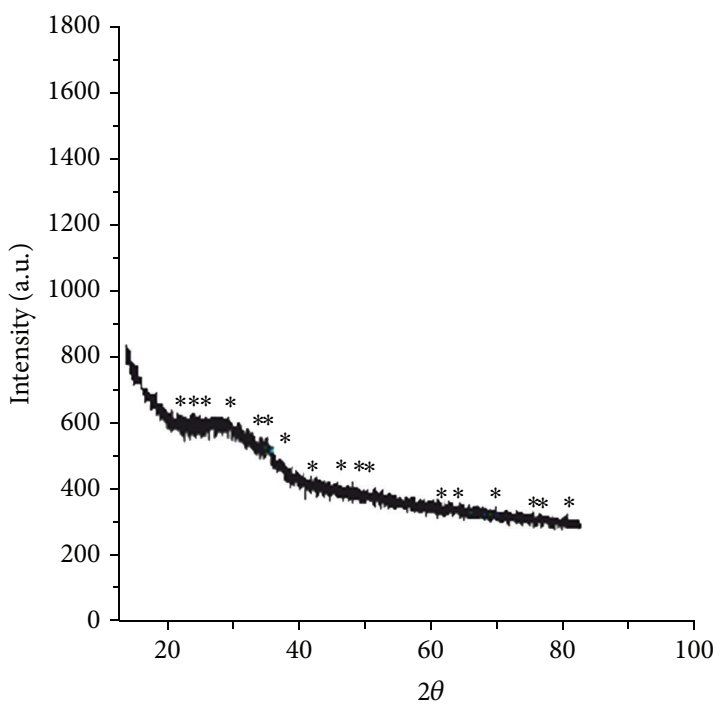

* Hydroxyapatite

FIGURE 5: X-ray diffraction pattern was obtained for the samples of HA.

speeds. In this case and at this scanning speed (1 s/line), the nanoparticles are stable to thermal effect. Furthermore, the spectroscopic mode provides a current that is stable in all the systems of tunneling. Alternatively, this analysis offers a better

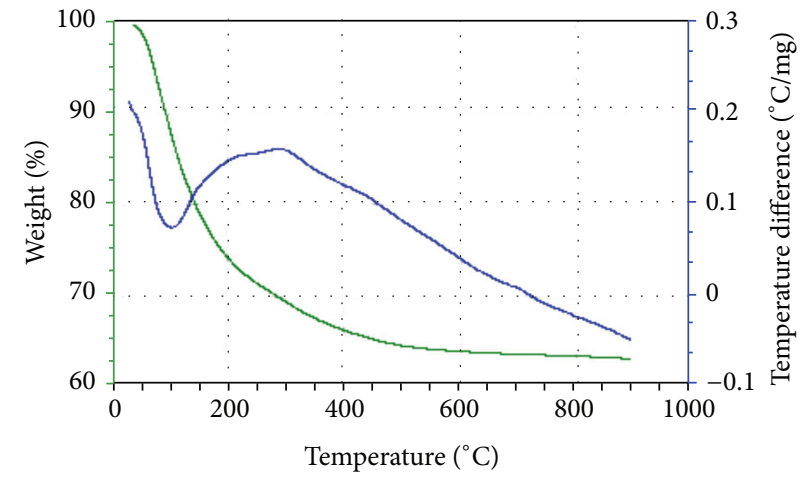

Figure 6: TGA-DTA analysis of the HA nanoparticles.

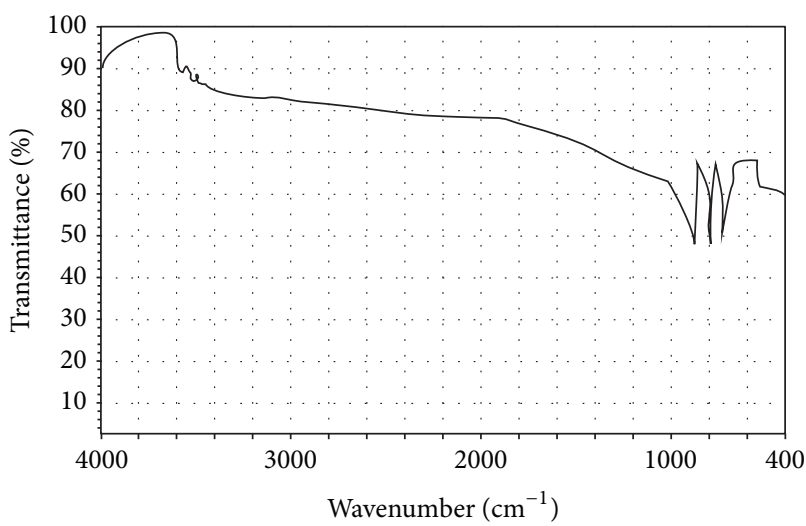

FIGURE 7: IR spectra of nanoparticles complex.

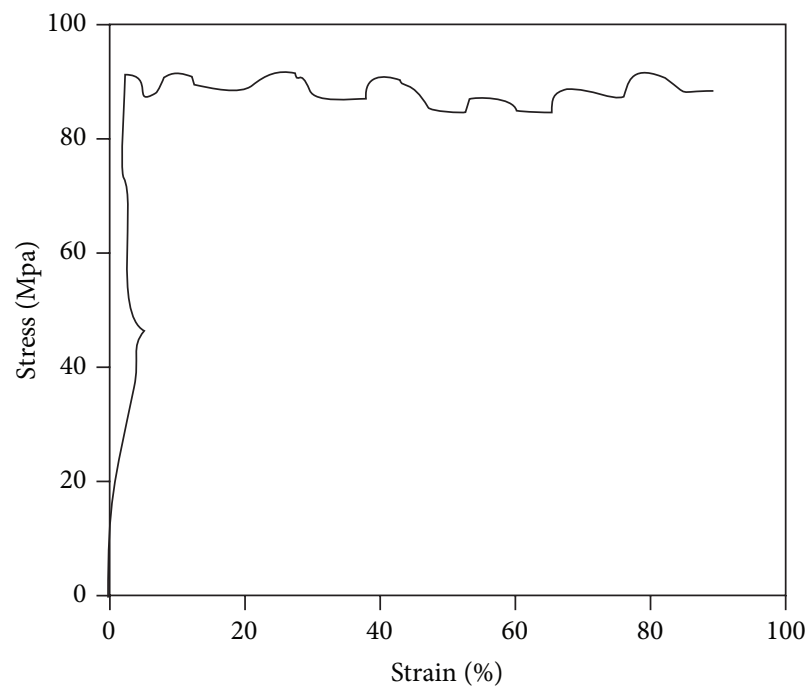

FIGURE 8: Stress-strain curve of nanoparticles.

understanding of how to achieve control of the synthesis of nanostructures based on materials self-assembly with polysaccharides network.

In Figure 4 that the particle size distribution in a range of $10-11 \mathrm{~nm}$ is observed. Approximately $47 \%$ of the nanoparticles obtained in this work are in the range of about $10 \mathrm{~nm}$ in 
Hydroxyapatite Linear polysaccharide

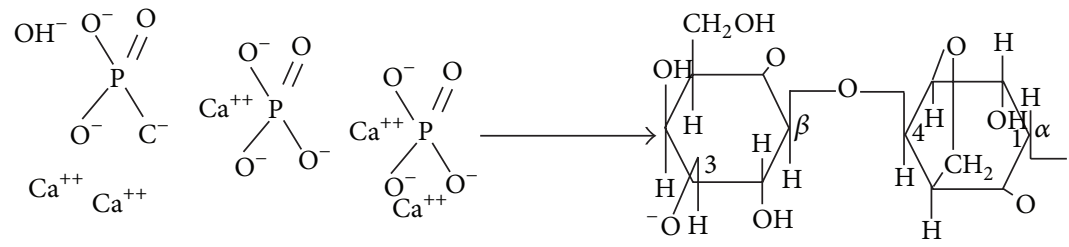

FIGURE 9: Interaction of hydroxyapatite and the linear polysaccharide.

diameter. The obtained particle size was done in the middle of the measurement of particle diameter and the number of particles found in electron microscopy images.

Some studies have reported particles size $(10-100 \mathrm{~nm})$ using mechanochemical process and surfactant-assisted ball milling technique $[16,17]$, but they have not reported the synthesis of nanoparticles with controlled size through networks of polysaccharide.

Figure 5 shows the diffraction patterns of the powders at $810^{\circ} \mathrm{C}$. In this plot, the sample is characterised by peaks at $22^{\circ}$, $23^{\circ}, 24.8^{\circ}, 28^{\circ}, 31^{\circ}, 32^{\circ}, 34^{\circ}, 40^{\circ}, 44^{\circ}, 45^{\circ}, 49.5^{\circ}, 50^{\circ}, 51^{\circ}, 52^{\circ}, 53^{\circ}$, $60^{\circ}, 62^{\circ}, 68^{\circ}, 72^{\circ}, 73^{\circ}$, and $79^{\circ}$, which corresponds to the HA. In this case, it is interesting that the phases of the precursors compounds can interact and be present as it is shown in the X-ray diffraction patterns. The characteristic peaks of hydroxyapatite were identified in the X-ray diffraction pattern (XRD). This pattern showed that the powder sample was perfectly crystalline and composed of HA. The degree of HA crystallinity was calculated by the following equation: $X c \approx 1-\left(V_{112-300} / I_{300}\right)$. In this equation, $X c$ is the degree of crystallinity, $V_{112-300}$ is the depth of the valley between the characteristic peaks corresponding to the planes of (112) and (300), and $I_{300}$ is the intensity of (300) planes. The sizes of the crystallites estimated from X-ray diffraction pattern using Scherrer equation and the crystallinity of HA phase were about $5 \mathrm{~nm}$ and $66 \%$ respectively.

Figure 6 shows the behavior of the HA with TGA-DTA method. The thermal gravimetric analysis (TGA) shows a weight loss in the temperature region $50-200^{\circ} \mathrm{C}$. The differential thermal analysis (DTA) shows exothermic peaks in the temperature region of $250-300^{\circ} \mathrm{C}$. The purpose of this test is to determine how the HA is affected by temperature.

The surface area of the sample was determined using the Brunauer-Emmett-Teller analysis (BET). The particle size was calculated from surface area, assuming spherical particles, following equation (3). DBET $=6000 /(\rho S)$, where DBET is the equivalent particle diameter in nanometers, $(\rho)$ the density of the particle in $\left(\mathrm{g} / \mathrm{cm}^{3}\right)$, and $(S)$ the specific surface area in $\left(\mathrm{m}^{2} / \mathrm{g}\right)$. We obtained from BET analysis the specific area as $130 \mathrm{~m}^{2} / \mathrm{g}$, so the average equivalent particle size is between 10 and $11 \mathrm{~nm}$.

The IR spectrum of the nanoparticles has been recorded on a Perkin-Elmer infrared spectrophotometer within the wavenumber range of $4000 \mathrm{~cm}^{-1}-400 \mathrm{~cm}^{-1}$. In Figure 7, the IR spectrum shows absorption peaks corresponding to the $\beta$ - $D$ galactopyranose. A band appears in the IR spectrum at $750 \mathrm{~cm}^{-1}$. Another band appears at $800 \mathrm{~cm}^{-1}$ and another one at $900 \mathrm{~cm}^{-1}$. This band appears due to the $\mathrm{C}-\mathrm{O}$ and $\mathrm{C}-$ $\mathrm{C}$ vibrations of the polysaccharides. On the other hand, the first indication for the formation of $\mathrm{HA}$ is in the form of stretching. The $\mathrm{O}-\mathrm{H}$ stretching bond is shown at $3575 \mathrm{~cm}^{-1}$ and $3550 \mathrm{~cm}^{-1}$ in this graphic which confirms the presence of hydroxyapatite powder. Upon compression the $(\mathrm{OH})$ band shifts to higher wavenumbers; however, on the librational mode exists an opposite effect. Theoretically the distance between two oxygen molecules is minimal when the pressure increases. In this manner the stretching of a hydrogen bond should decrease and thus all frequencies should increase; furthermore in the apatites the moving of the $\mathrm{OH}$ groups is very complicated because they are always interacting on the $c$ axis. This means that vibrations are not found in parallel alignment; these differences may be due to the effects of loads developed on the surface of the hydroxyapatite which would lead to the fact that the longitudinal and transverse frequency is increased with the increase in the intensity of absorption affecting principally the $\mathrm{OH}$ stretching and $\mathrm{O}^{2-}$ displacements.

In Figure 8, stress-strain curve shows the sample of nanoparticles; the compounds have a strain of $90 \%$ and a stress of 91 Mpa.

We think that compounds that have high flexion due to the $\left(\mathrm{Ca}^{++}\right)$group can interact with the $(-\mathrm{OH})$ of the polymers chains of galactopyranose, (Figure 9). In this context the hypothesis to explain why the curve is limited to $90 \%$ may be that the Ca present in the compounds has not clearly defined its yield point or yield strength (elastic limit.). Additionally, this would be accompanied by a movement of dislocations that it would be very difficult to detect and determine their elastic limit. In this way this polysaccharide would be playing an important role in Hooke's law. Hooke's law is not met because the stress is proportional to strain and does not follow a straight line in the curve. This in no way would be negative for the production of nanoparticles, because the behavior of the stress-strain curves also depends on the nature of each material.

\section{Conclusions}

This paper reports the formation of hydroxyapatite nanoparticles in a range of $10-11 \mathrm{~nm}$ in diameter at a low cost as 
an easy route for the fabrication of nanostructure materials. These materials can be used in diverse areas as materials sciences, bioengineering, nanomaterials for medicine, and electronic systems. The results of the X-ray confirmed that the compounds obtained show crystalline phases of the metallic precursors at $810^{\circ} \mathrm{C}$. Furthermore, the characteristics of materials are important so that the compounds can be functionalized on the surface of organic molecules and with this method it would be possible to analyze several events as $\mathrm{pH}$, temperature, and formation of a polymeric porous network.

\section{Acknowledgments}

The author would like to thank Daniel Lardizabal and Enrique Torres for their technical assistance.

\section{References}

[1] L. L. Hench, "Bioceramics: from concept to clinic," Journal of the American Ceramic Society, vol. 74, no. 7, pp. 1487-1510, 1991.

[2] R. Kumar, K. Prakash, P. Cheang, and K. Khor:, "Temperature driven morphological changes of chemically precipitated hydroxyapatite nanoparticles," Langmuir, vol. 20, no. 13, pp. 51965200, 2004.

[3] S. Sadasivan, D. Khushalani, and S. Mann:, "Synthesis of calcium phosphate nanofilaments in reverse micelles," Chemistry of Materials, vol. 17, no. 10, pp. 2765-2770, 2005.

[4] P. L. Brannon and J. O. Blanchette, "Nanoparticle and targeted systems for cancer therapy," Advanced Drug Delivery Reviews, vol. 56, no. 11, pp. 1649-1659, 2004.

[5] V. Wagner, A. Dullaart, A. K. Bock, and A. Zweck, "The emerging nanomedicine landscape," Nature Biotechnology, vol. 24, no. 10, pp. 1211-1217, 2006.

[6] C. Jiyong, J. G. Wolke, and C. K. Groot, "Microstructure and crystallinity in hydroxyapatite coatings," Biomaterials, vol. 15, no. 5, pp. 396-399, 1994.

[7] D. M. Liu, H. M. Chou, and J. D. Wu, "Plasma-sprayed hydroxyapatite coating: effect of different calcium phosphate ceramics," Journal of Materials Science, vol. 5, no. 3, pp. 147-153, 1994.

[8] A. Siddharthan, S. K. Seshadri, and T. S. Sampath Kumar, "Rapid synthesis of calcium deficient hydroxyapatite nanoparticles by microwave irradiation," Trends in Biomaterials and Artificial Organs, vol. 18, no. 2, pp. 110-113, 2005.

[9] Y. Sun, G. Guo, D. Tao, and Z. Wang, "Reverse microemulsiondirected synthesis of hydroxyapatite nanoparticles under hydrothermal conditions," Journal of Physics and Chemistry of Solids, vol. 68, no. 3, pp. 373-377, 2007.

[10] Y. Liu, J. Rong, A. Zhou et al., "Study on high precision and super-slow speed feeding table for micro-EDM machining," Key Engineering Materials, vol. 339, pp. 332-336, 2007.

[11] G. D. Prestwich, D. M. Marecak, J. F. Marecek, K. P. Vercruysse, and M. R. Ziebell, "Controlled chemical modification of hyaluronic acid: synthesis, applications, and biodegradation of hydrazide derivatives," Journal of Controlled Release, vol. 53, no. 1-3, pp. 93-103, 1998.

[12] A. Sinha and G. Avijit, "Biomimetic patterning of polymer hydrogels with hydroxyapatite nanoparticles," Materials Science and Engineering C, vol. 29, no. 4, pp. 1330-1333, 2009.
[13] S. B. Tiwari and M. M. Amiji, "Improved oral delivery of paclitaxel following administration in nanoemulsion formulations," Journal of Nanoscience and Nanotechnology, vol. 6, no. 9-10, pp. 3215-3221, 2006.

[14] W. Weng and J. L. Baptista, "Sol-gel derived porous hydroxyapatite coatings," Journal of Materials Science, vol. 9, no. 3, pp. 159-163, 1998.

[15] X. Lu and Y. Leng, "Theoretical analysis of calcium phosphate precipitation in simulated body fluid," Biomaterials, vol. 26, no. 10, pp. 1097-1108, 2005.

[16] S. Ohara, K. Sato, Z. Tan, H. Shimoda, M. Ueda, and T. Fukui, "Novel mechanochemical synthesis of fine FeTiO3 nanoparticles by a high-speed ball-milling process," Journal of Alloys and Compounds, vol. 504, no. 1, pp. L17-L19, 2010.

[17] M. Yue, Y. P. Wang, N. Poudyal, C. B. Rong, and J. P. Liu, "Preparation of Nd-Fe-B nanoparticles by surfactant-assisted ball milling technique," Journal of Applied Physics, vol. 105, no. 7, Article ID 07A708, 2009. 

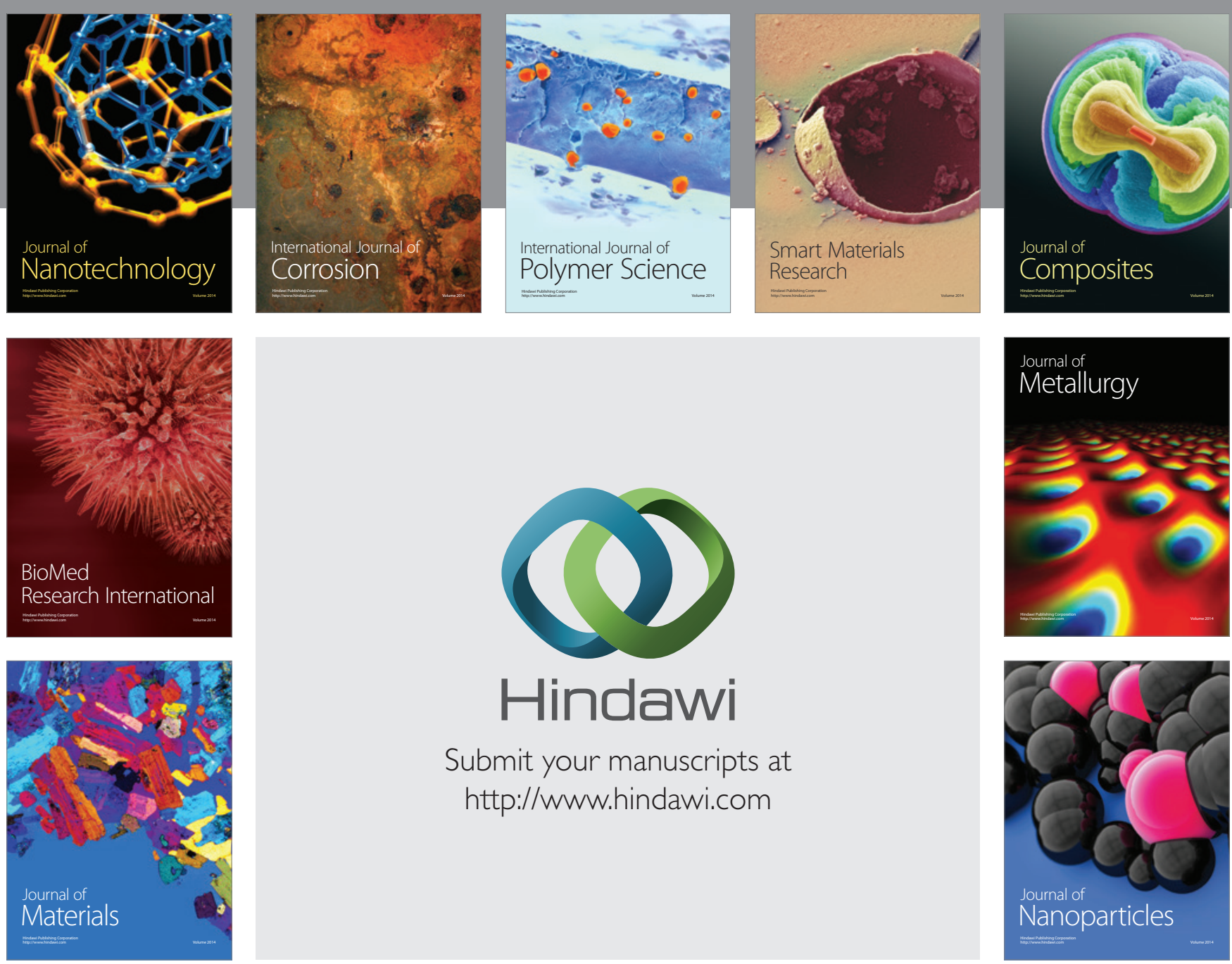

Submit your manuscripts at http://www.hindawi.com
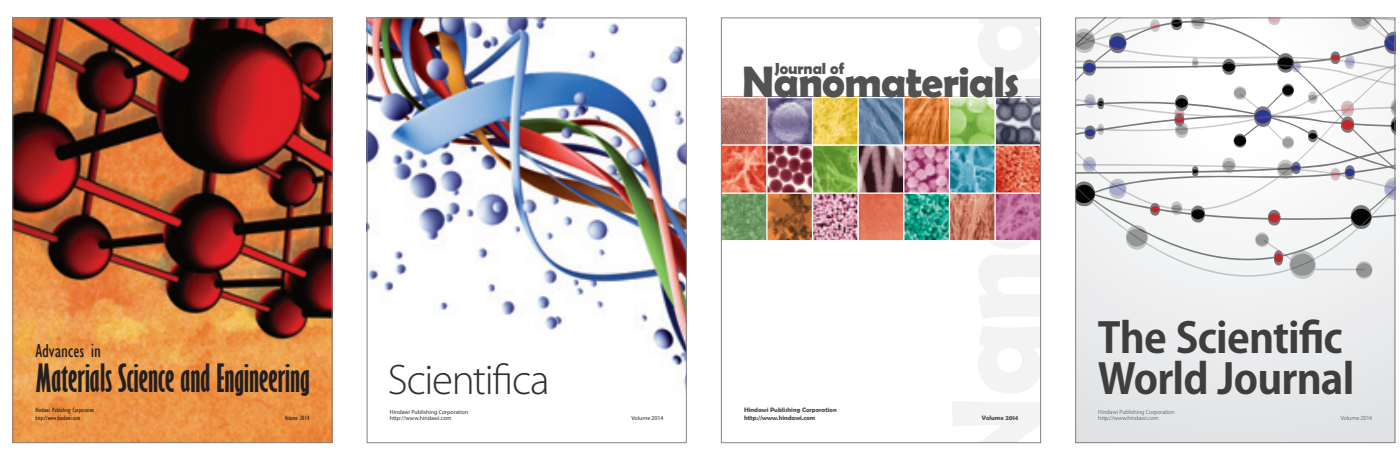

\section{The Scientific World Journal}
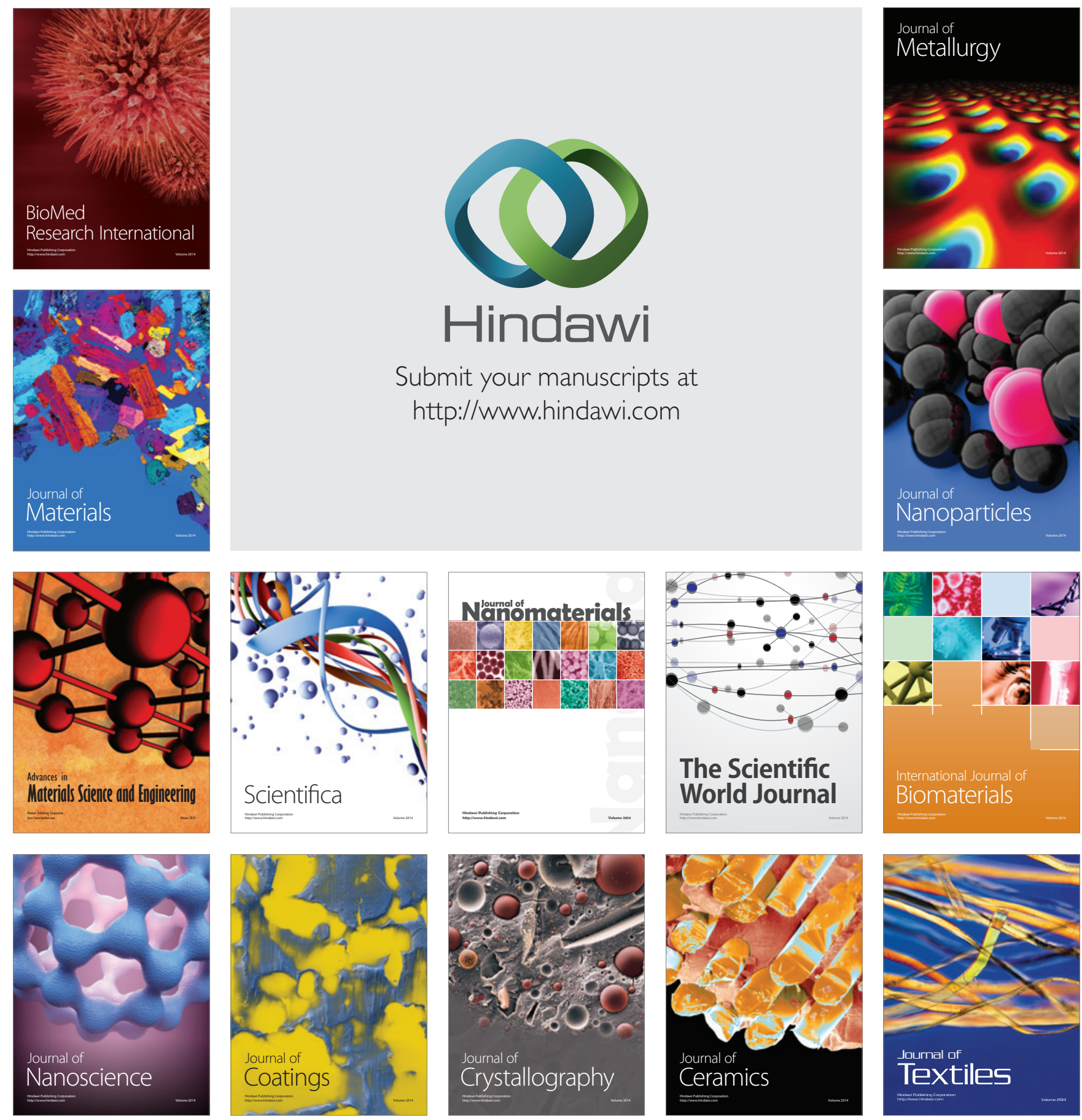